\title{
ELECTROWEAK MEASUREMENTS AT THE TEVATRON
}

\author{
JOSE E. GARCIA \\ On behalf of the CDF and DØ Collaborations, \\ INFN Sezione di Pisa, \\ Largo B. Pontecorvo 3, \\ 56100 Pisa, Italy \\ E-mail: Jose.Enrique.Garcia@pi.infn.it
}

\begin{abstract}
Recent Electroweak measurements by the CDF and $D \varnothing$ collaborations in $p \bar{p}$ collisions at $\sqrt{s}=1.96 \mathrm{TeV}$ are presented here. Measurements of $\mathrm{W}, \mathrm{Z}$ and diboson production cross sections as well as $\mathrm{W}$ asymmetry using integrated luminosities up to $800 \mathrm{pb}^{-1}$ are reviewed. Limits on triple gauge anomalous couplings on diboson production are discussed elsewhere ${ }^{1}$.
\end{abstract}

\section{Introduction}

The Tevatron accelerator is currently running at a high center of mass energy $(\sqrt{s}=1.96 \mathrm{TeV})$ and high luminosity $\left(\sim 10^{32} \mathrm{~cm}^{-2} \mathrm{~s}^{-1}\right)$. Large quantities of $W$ and $Z$ bosons are produced weekly in the accelerator collisions. $\mathrm{W}$ and $\mathrm{Z}$ production is precisely predicted by the Standard Model (SM). Experimental measurements of the boson production cross sections provide stringent tests of the SM and aid in understanding detector performance. Measuring the $\mathrm{W}$ charge asymmetry provides information on the $d$ and $u$ quarks inside the proton. Results help to further constrain Parton Distribution Functions (PDF).

Diboson production implies triple gauge couplings which are very sensitive to new physics. Thus measuring diboson cross sections is a way to probe for new physics.

\section{Inclusive cross section measurements}

In hadronic colliders like the Tevatron, the detection of $\mathrm{W}$ and $\mathrm{Z}$ bosons through their hadronic decay is overwhelmed by the QCD background. Their decay into leptons is the cleanest way to identify them. The signature of these decays is a high energy lepton with $P_{T}>20 \mathrm{GeV}$ observed in 
the fiducial part of the detector volume. For $\mathrm{W}$ candidates the additional requirement is large missing transverse energy $\left(E_{T}>25 \mathrm{GeV}\right)$ and for $\mathrm{Z}$ candidates a second electron with opposite charge where the resulting mass of the dilepton pair lies within the $Z$ window.
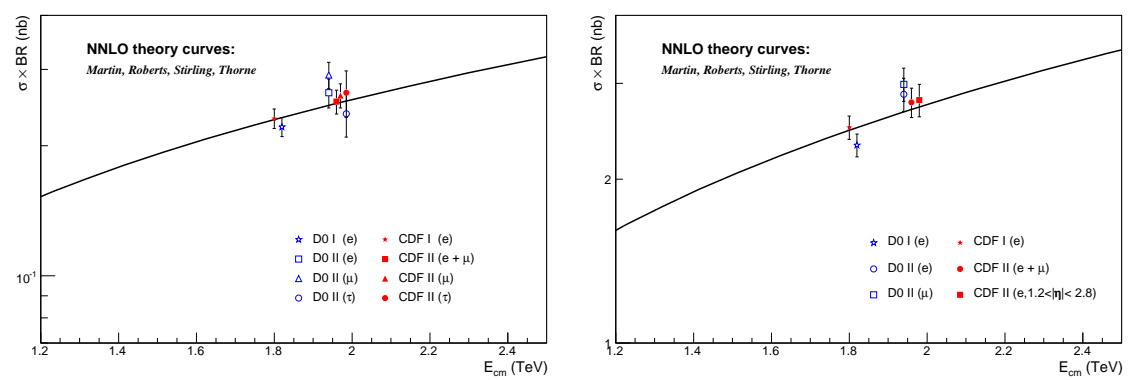

Figure 1. Summary of CDF and $D \varnothing \mathrm{W}$ cross section measurements in all three leptonic decay channels.

The $\mathrm{W}$ and $\mathrm{Z}$ cross sections have been measured by the $\mathrm{CDF}$ and $D \emptyset$ in different lepton channels. Figure 1 shows a summary of the measurements in all leptonic decay modes. Measurements are in agreement with the NNLO calculations (black line). At present, precision is limited by systematic uncertainties: lepton identification $(1-3 \%)$, PDF uncertainties $(1-2 \%)$ and the dominant uncertainty, luminosity, with a $6 \%$ contribution.

\subsection{W cross section measurement in the forward region}

A new measurement has been performed at CDF taking advantage of the forward calorimeter and the large coverage of the tracking system. The $\mathrm{W}$ cross section has been measured using electrons in the forward region. W boson candidates are selected by requiring a high $E_{T}$ electron $\left(E_{T}>20\right.$ $\mathrm{GeV}$ ) in the region $1.2<|\eta|<2.8$ and large missing transverse energy coming from the neutrino $\left(E_{T}>25 \mathrm{GeV}\right)$. Further cuts are applied to the electron candidate to increase sample purity. The energy deposit in the electromagnetic calorimeter is required to match a track from the tracking system and the electron has to be isolated. The $\mathrm{W}$ cross section value obtained is $2.796 \pm 0.013$ (stat. $)_{-0.090}^{+0.095}$ (sys.) \pm 0.168 (lum.) $n b$. The result is in agreement with CDF measurements in the central region ${ }^{2}$ and with theoretical estimates. 



Figure 2. Measurement of $W \rightarrow \mu \nu$ asymmetry (left) and with CP folding (right). With the data (points) the CTEQ6.1 PDF $\pm 1 \sigma$ band and the central MRST02 PDF value (curve) is shown.

\section{W charge asymmetry}

Cross section measurements are limited by the PDF uncertainties. More precise measurements can be obtained with a better knowledge of the parton distribution functions. One way to obtain additional information on PDFs is through the study of the $\mathrm{W}$ charge asymmetry.

$\mathrm{W}$ bosons at the Tevatron are mainly produced through annihilation of $u$ and $d$ quarks. As the $u$ quark carries on average a higher fraction of the proton momentum $(x)$, the $W^{+}$tends to be boosted in the proton direction, while the $W^{-}$is boosted in the anti-proton direction. This leads to a forward-backward charge asymmetry for the $W$ boson production. However, since it is not possible to measure the neutrino longitudinal momentum, the rapidity of the $\mathrm{W}$ bosons can not be reconstructed directly from the $\mathrm{W}$ candidate. The quantity that it is measured is:

$$
A\left(\eta_{\ell}\right)=\frac{d \sigma\left(\ell^{+}\right) / d \eta_{\ell}-d \sigma\left(\ell^{-}\right) / d \eta_{\ell}}{d \sigma\left(\ell^{+}\right) / d \eta_{\ell}+d \sigma\left(\ell^{-}\right) / d \eta_{\ell}} \propto \frac{d(x)}{u(x)}
$$

where $\eta_{\ell}$ is the lepton pseudo-rapidity. The observed asymmetry is a convolution of the $\mathrm{W}$ asymmetry with the $V-A$ couplings. This measurement is sensitive to the ratio of the $u$ and $d$ momentum fractions within the proton. $D \varnothing$ has also measured this asymmetry in the muon channel utilizing its large muon coverage $\left(\left|\eta_{\mu}\right|<2.0\right)$. Figure 2 shows this asymmetry corrected for charge misidentification and background contribution.

\section{Diboson Production: $W W$ and $W Z$}

Studying diboson production provides an important test of the SM. Non-SM contributions to triple gauge couplings in diboson production may increase 
Table 1. Last results for diboson production at the Tevatron.

\begin{tabular}{ccccc}
\hline & \multicolumn{2}{c}{$C D F$} & \multicolumn{2}{c}{$D \varnothing$} \\
& $\mathcal{L}\left(p b^{-1}\right)$ & $\sigma(p b)$ & $\mathcal{L}\left(p b^{-1}\right)$ & $\sigma(p b)$ \\
\hline$W(\ell \nu) W(\ell \nu)$ & 825 & $13.6 \pm 2.8($ stat $)$ & 252 & $13.8_{-3.8}^{+4.3}($ stat $)$ \\
$W(\ell \nu) Z(\ell \ell)$ & 825 & $<6.34$ & 320 & $<13.3$ \\
\hline
\end{tabular}

the production cross sections significantly. Here we report on measurements of production cross sections for $W W$ and $W Z$.

The first evidence for $\mathrm{W}$ pair production was observed by CDF in $p \bar{p}$ collisions at $\sqrt{s}=1.8 \mathrm{TeV}^{5}$. With increasing statistics it is now possible to measure the $W W$ cross section with a significant precision. Due to the large QCD background, diboson production has been studied mainly through the leptonic decay channels. Candidate events are required to have two well identified leptons with opposite charge and large missing transverse energy. Table 1 shows the latest results obtained by CDF and $D \emptyset$. The values are in good agreement with the theoretical NLO calculation of $12.4 \pm 0.8 p b^{6}$.

$W Z$ production proceeds through the $W W Z$ vertex which is not available at $e^{+} e^{-}$colliders. At the Tevatron $W Z$ production has been studied by looking at leptonic decays. The fully leptonic decay presents a clean tri-lepton final state. We select events with three leptons and at least 25 $\mathrm{GeV}$ of missing transverse energy associated with the neutrino from the $W$ boson. Two of the leptons must have opposite sign and an invariant mass between 76 and $106 \mathrm{GeV}$. Table 1 also shows the limits that CDF and $D \varnothing$ place on $W Z$ production which are consistent with the NLO calculation for this process $(3.65 \pm 0.26 p b)$.

\section{Acknowledgments}

This work has been partially supported by the European Community's Human Potential Programme under contract HPRN-CT-2002-00292.

\section{References}

1. J. Sjolin, arXiv:hep-ex/0605094.

2. D. Acosta et al., Phys. Rev. Lett. 94091803 (2005).

3. D. Acosta et al., Phys. Rev. D 71051104 (2005).

4. CDF and DØ Collaborations, Phys. Rev. D 70092008 (2004).

5. F. Abe et al., Phys. Rev. Lett. 784536 (1997).

6. J.M. Campbell and R.K. Ellis, Phys. Rev. D 60113006 (1999). 